Neurosurg Focus 13 (1):Introduction, 2002 Click here to return to Table of Contents

\title{
Spondylolisthesis: introduction and illustrative cases
}

\author{
ROBERT F. HEARY, M.D. \\ Department of Neurological Surgery, University of Medicine and Dentistry of New Jersey, New Jersey \\ Medical School, Newark, New Jersey
}

The current issue of Neurosurgical Focus deals with spondylolisthesis. This common spinal disorder has numerous classifications and schemes for management and treatment. As a general rule, spondylolisthesis refers to a translational movement of one vertebra with respect to its adjacent vertebra. The involved level is identified by naming each of the included vertebrae. In the most common circumstance, the superior vertebra translates forward on the inferior vertebra, causing an anterolisthesis. Occasionally, the superior vertebra may move backward with respect to the inferior vertebra, and this is referred to as a retrolisthesis. Specifying the slippage is accomplished by defining the relationship of the superior vertebra to the inferior vertebra. If no specification is made, the generic term spondylolisthesis refers to an anterolisthesis. Traditional classifications of spondylolisthesis include the categories of degenerative, isthmic or lytic, congenital, and dysplastic. As the majority of patients with spondylolisthesis who come to a neurosurgeon's office have either the degenerative or isthmic variety, this issue of Neurosurgical Focus covers these two categories. Classically, isthmic spondylolisthesis is seen at the L5-S1 level and degenerative spondylolisthesis is most often seen at the L4-5 level. Degenerative spondylolisthesis is seen less frequently at other levels in the lumbar spine.

Treatment options for spondylolisthesis are plentiful. Variations exist from minimally invasive techniques to major spinal reconstructions. In the rapidly evolving field of spinal neurosurgery, percutaneous techniques have been developed that allow for the placement of pedicle screws and interbody devices. As these are new approaches, long-term results from minimally invasive spinal reconstruction are not yet available. The more traditional approaches to the treatment of spondylolisthesis have included posterior surgery, anterior surgery, or combined anterior-posterior, referred to as "AP or $360^{\circ}$ " surgery. These open surgical procedures may include reduction of the subluxation or in situ treatment of the disorder. There are an assortment of methods that are acceptable for the treatment of spondylolisthesis, which may vary based on the age of the patient, the type of abnormality, and the experience and comfort of the treating surgeon.

In this issue of Neurosurgical Focus, Dr. Ganju addresses the topic of isthmic spondylolisthesis. The specific issues relating to whether reduction is necessary and/or safe are reviewed by Drs. Rengachary and Balabhandra. Major open spinal reconstructions are discussed by Drs. Heary and Bono. Drs. Palmer and Turner provide an interesting new insight into the less invasive treatment of spondylolisthesis. Along with this introduction, I have included two isolated illustrative case presentations to demonstrate alternative methods of treating spondylolisthesis.

I would like specifically to thank the reviewers who have generously donated their time in the peer-review process. For this issue of Neurosurgical Focus, the peer-review panel consisted of: Joseph T. Alexander, M.D., Wake Forest University; Michael W. Groff, M.D., Indiana University; Timothy C. Ryken, M.D., the University of Iowa; and Gregory R. Trost, M.D., the University of Wisconsin. 


\section{ILLUSTRATIVE CASE 1: THE MANAGEMENT OF HIGH-GRADE ISTHMIC SPONDYLOLISTHESIS}

This 41-year-old woman presented with a history of low-back pain and had had poor posture since adolescence. She complained of progressive worsening of her back pain for a 5-year period, which was unresponsive to conservative therapy that included both physical therapy and chiropractic treatment. She denied motor weakness. She did note bilateral paresthesias in the L-5 and S-1 dermatomes. She denied any bowel or bladder symptoms. She was in otherwise excellent health.

On physical examination, the patient had normal power in her lower extremities and diminished pinprick sensation in the L5-S1 dermatomes. Reflexes were normal at the quadriceps muscles and absent at the ankles bilaterally. Her posture was noteworthy for a marked flexion at the hips with prominence of the sacrum. On plain film radiographs, a high-grade (Grade IV) spondylolisthesis was present. There was no motion on dynamic flexion-extension lateral radiographs. A magnetic resonance (MR) imaging study demonstrated the high-grade slippage and marked narrowing of the L-5 foramina bilaterally.

The patient chose to undergo elective surgery for a decompression with fusion and stabilization procedure. Due to the chronicity of the problem and the high grade of the subluxation, the preoperative plan called for an in situ decompression with no attempt at reduction. A wide posterior removal of the spinous process and laminae bilaterally (called the Gill fragment) was performed.

A circumferential fusion was then performed. Posteriorly, a bilateral lateral fusion between the transverse processes of L4-5, and the sacral alae with autologous iliac

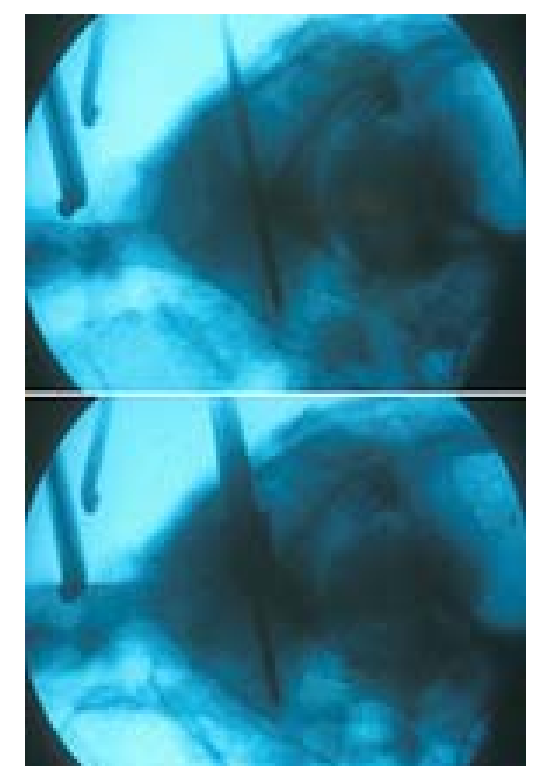

Fig. 1. Plain film radiographs (lateral view). Upper: High-grade L5-S1 spondylolisthesis seen on intraoperative fluoroscopic image; the Steinman pin has entered the dorsal aspect of S-1 and traversed through the S-2 and L-5 vertebrae. Lower: The intraoperative fluoroscopic image showing the $14-\mathrm{mm}$ reamer drilling a channel over the previously placed Steinman pin.

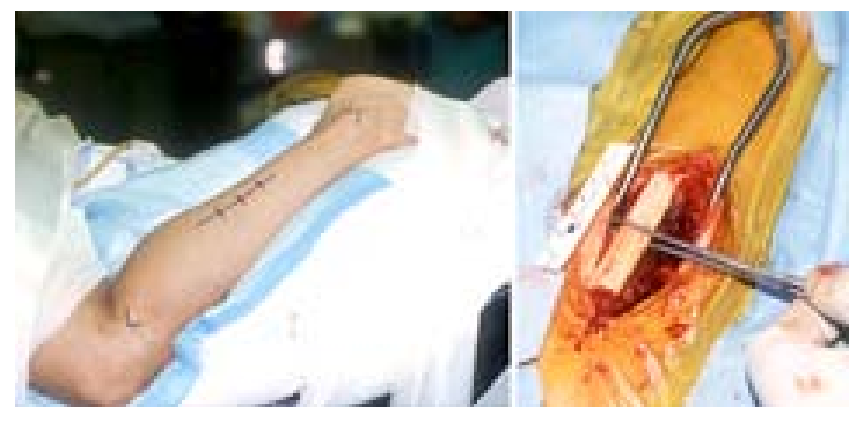

Fig. 2. Intraoperative photographs. Left: Demonstration of the position for obtaining an autologous fibula graft. Right: Image of a 7-cm piece of bone, which was harvested from the midportion of the fibula.

crest grafts was placed. The anterior approach included placement of a Steinman pin from the dorsal aspect of the S-1 vertebra through the sacrum and then through the L-5 vertebra to its anterosuperior most point (Fig. 1 upper). With gentle retraction of the left $\mathrm{S}-1$ nerve root and working through the axilla of the nerve root, a 14- $\mathrm{mm}$ reamer was then used to drill a path over the Steinman pin (Fig. 1 lower).

Via a separate incision, the left fibula region was prepared and draped for harvesting an autologous fibular graft (Fig. 2 left). This graft was $7 \mathrm{~cm}$ long and care was taken to harvest the graft from the midportion of the fibula (Fig. 2 right). At least $7 \mathrm{~cm}$ must be preserved between the graft and the joints above and below to avoid the delayed morbidity of instability.

The autologous fibular graft was gently impacted into the tract, which had been previously reamed until the graft was countersunk into the tract at the axilla of the left S-1 nerve root (Fig. 3 left). Following graft placement, stabilization was accomplished with pedicle screws placed in

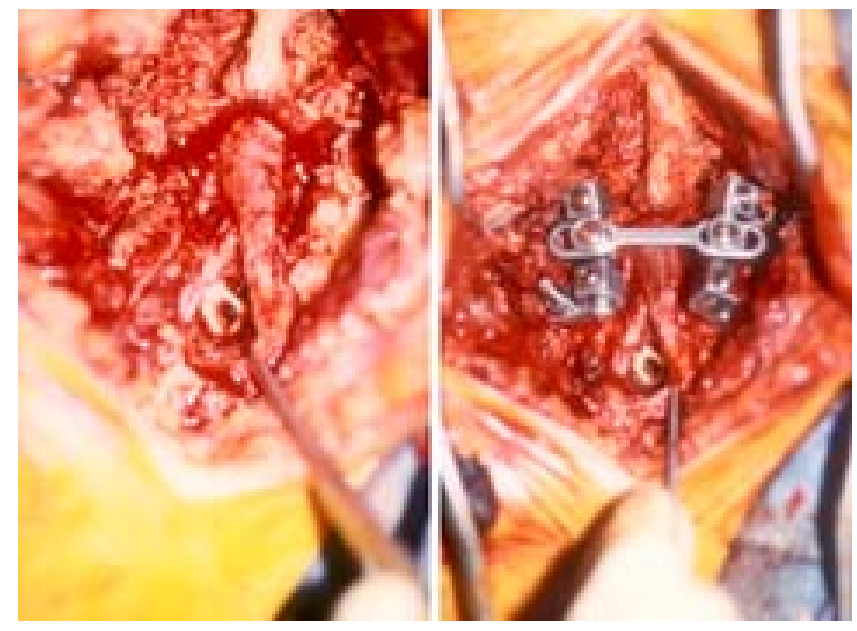

Fig. 3. Intraoperative photographs. Left: Image of the autologous fibular graft after it was impacted through the axilla of the left $\mathrm{S}-1$ nerve root; the graft is countersunk $2 \mathrm{~mm}$ beneath the dorsalmost aspect of the S-1 vertebra. Right: Image of the final construct, which demonstrates pedicle screws in L-4 and S-1 bilaterally, the fibular graft, and a cross-connector between the two rods. 


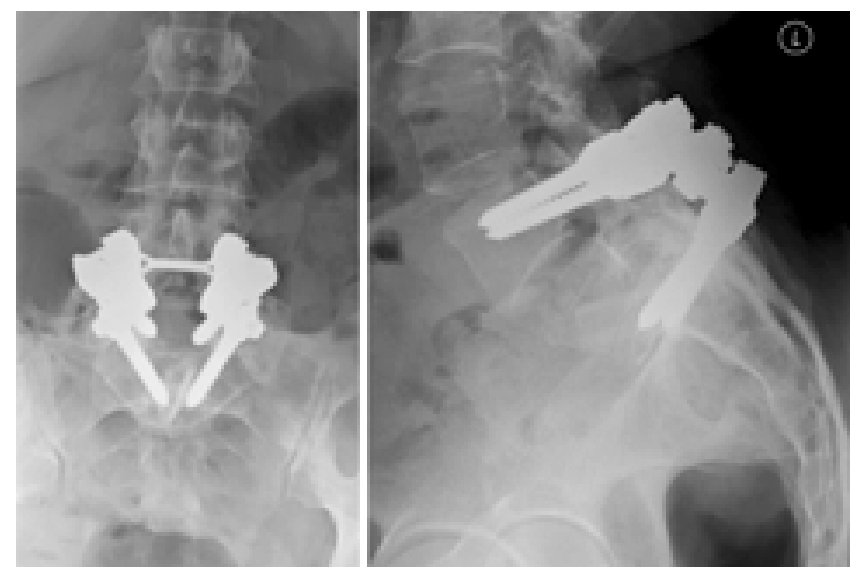

Fig. 4. Plain film radiographs. Anteroposterior (left) and lateral (right) views taken more than 5 years after surgery, demonstrating complete incorporation of the fusion construct; the fibular graft is visible.

the L-4 and S-1 pedicles bilaterally. Rods were cross-connected for rotational stability (Fig. 3 right).

At long-term 6-year follow-up examination the patient had a solid radiographically demonstrated fusion. Anteroposterior and lateral (Fig. 4) radiographs demonstrate a solid construct with the fibula well incorporated. The patient is currently pain free with no neurological deficits. She is able to perform all activities of daily living with no restrictions.

\section{ILLUSTRATIVE CASE 2: MANAGEMENT OF LOW-GRADE ISTHMIC SPONDYLOLISTHESIS}

This 42-year-old man, a contractor in the construction industry, presented with a 2-year history of severe pain that radiated to the right hip and lower extremity. The pain

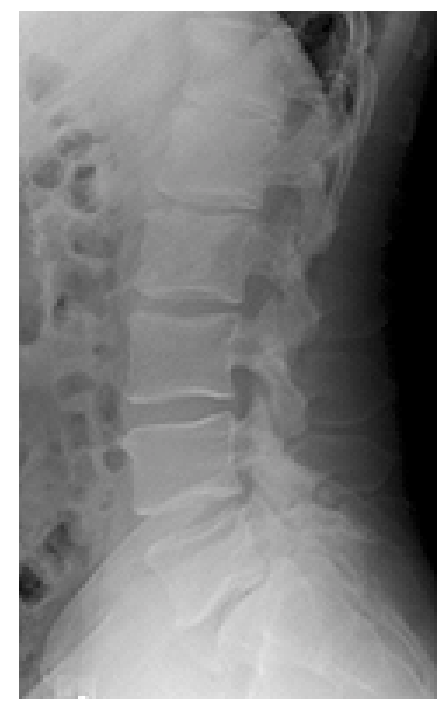

Fig. 5. Plain film radiograph (lateral view) revealing a Grade I-II isthmic (lytic) spondylolisthesis.

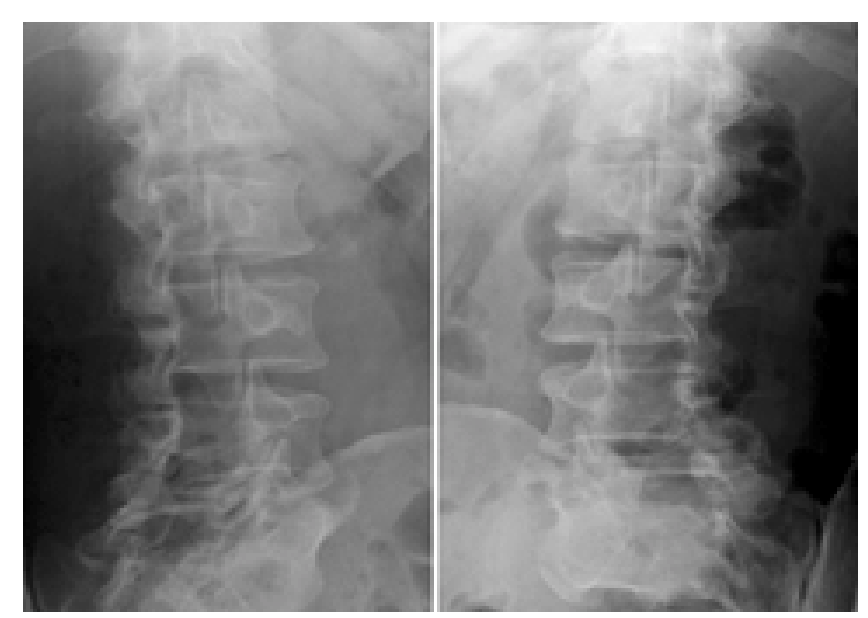

Fig. 6. Plain film radiographs (right and left oblique views) demonstrating the absence of a pars interarticularis of L-5 bilaterally (referred to as a loss of the "neck of the Scottie dog" in this projection).

had progressed significantly over a 3-month period prior to his evaluation and had become intractable. There was no response to conservative therapy. He denied muscle weakness or any bowel or bladder symptoms. He did complain of right L-5 dermatomal region tingling. He was in otherwise excellent health.

On physical examination, motor power was intact. Sensory examination was noteworthy for diminished pinprick perception in the right L-5 dermatome. Lower-extremity deep tendon reflexes were normal and straight-leg raising was negative bilaterally. Plain film radiographs revealed a low-grade isthmic spondylolisthesis (Fig. 5). The subluxation was associated with bilateral spondylolysis of L-5 (absence of pars interarticularis) (Fig. 6). There was no motion on dynamic flexion-extension lateral radiographs. An MR imaging study demonstrated the spondylolisthetic slippage as well as degenerative changes in the L5-S1 disc, which appeared black on $\mathrm{T}_{2}$-weighted MR images (Fig. 7).

The patient chose to undergo elective surgery. The goal of surgery was to decompress and then reduce the slippage to an anatomical alignment, followed by fusion and stabilization. The procedure performed was a wide decompression with removal of the Gill fragment. Dorsal bone removal included the spinous process of L-5, the L-5 laminae bilaterally, and the inferior facets of L-5 bilaterally. A very aggressive discectomy was then performed to prepare for an eventual interbody fusion as well as to facilitate the attempt at reduction of the slippage.

Reduction was accomplished with the use of instrumentation. Pedicle screws were placed into L4 -5 and S-1 bilaterally. The screws at L-5 had elongated necks for the reduction procedure. Plates were "short-bent" and placed over the three screw heads. A distractor was placed between the screws at L-4 and S-1. Following distraction, the L-5 nut was tightened, which elevated the screw and the L-5 vertebral body. During the reduction, the L-5 nerve roots were directly visualized to confirm that no nerve root compression was occurring. Fluoroscopy revealed that an anatomical reduction was achieved. 


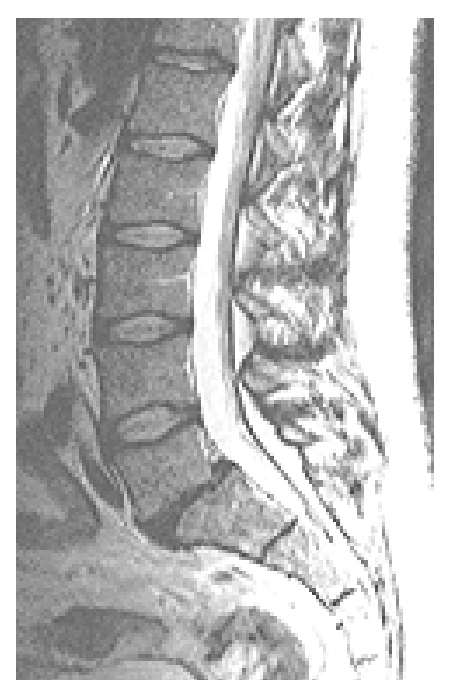

Fig. 7. A $\mathrm{T}_{2}$-weighted MR image demonstrating a loss of normal disc signal in the L5-S1 interspace with collapse of the height as well; low-grade spondylolisthesis is evident.

After the reduction was completed, a posterior lumbar interbody fusion was performed in which morselized autograft bone and allograft bone wedges were used. Distraction between the L-5 and S-1 screws was performed separately to allow each posterior lumbar interbody fusion wedge to be placed. After the wedge was positioned, release of the distraction allowed for compression of the graft. After this maneuver was completed bilaterally, bilateral lateral fusions were performed using autograft bone between the transverse processes of L-5 and the sacral alae.

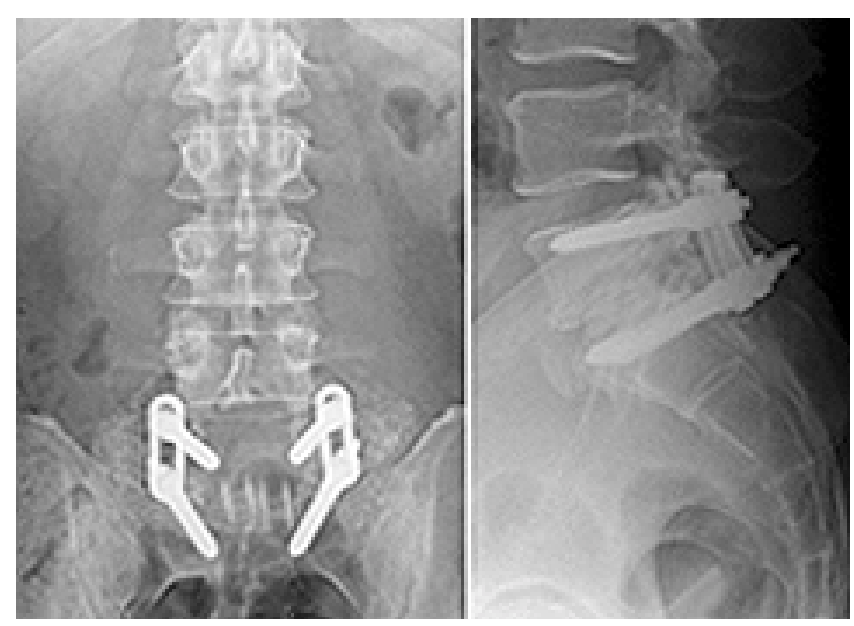

Fig. 8. Postoperative plain film radiographs. Left: The anteroposterior view shows good alignment with two structural allograft wedges in the interbody position; a generous amount of bone graft is visualized in the lateral regions overlying the L-5 transverse processes and the sacral alae bilaterally. Right: The anatomical reduction of the L5-S1 spondylolisthesis is well visualized on the lateral view.

At this point, the plate was removed on the left side and the L-4 pedicle screw was withdrawn. A short plate was then used to secure the L-5 screw to the S-1 screw. The identical procedure was then performed on the right hand side.

Postoperative radiographs have demonstrated an anatomical reduction of the L5-S1 spondylolisthesis (Fig. 8). At follow-up examination, the patient is pain free with no restrictions. 